\title{
Prediction of Aircraft Dynamic Stability Derivatives Using Time-Spectral Computational Fluid Dynamics*
}

\author{
Koji MIYAJI $^{1 \dagger}$ and Yu YosHIDA ${ }^{2)}$ \\ ${ }^{1)}$ Faculty of Engineering, Yokohama National University, Yokohama, Kanagawa 240-8501, Japan \\ ${ }^{2)}$ College of Engineering Science, Yokohama National University, Yokohama, Kanagawa 240-8501, Japan
}

\begin{abstract}
In this study, the dynamic stability derivatives of an aircraft model are calculated using CFD for the forced-pitch oscillation. The time-spectral, or reduced-frequency, method has been developed for RANS simulations on unstructured grids. It achieves faster computations than the time-marching method for periodically unsteady flows. The efficiency and accuracy of the method are first validated through comparisons with the transonic experiment of a pitching LANN wing. Next, the longitudinal dynamic-stability derivatives of a simplified aircraft model are calculated. Dependency of the damping-in-pitch and oscillatory longitudinal stability on the Mach number agreed reasonably well with the experimental results. Both the instantaneous flow field and frequency characteristics obtained directly from the time-spectral results are discussed to determine the effect of Mach number on the stability derivatives.
\end{abstract}

Key Words: CFD, Unstructured Grids, Aircraft Dynamic Stability

\section{Introduction}

The calculation of dynamic stability derivatives is an indispensable factor in considering the stability and maneuverability of aircraft. Attempts to obtain the dynamic derivatives through wind tunnel testing have a long history, and in many cases, two primary methods are the free oscillation method for measuring the displacement of motion at a limited degreeof-freedom of motion, and the forced oscillation method for measuring the force and moment acting on the model under a prescribed motion. ${ }^{1-3)}$ Meanwhile, with the improvement of CFD analysis capability, the prediction of dynamic stability derivatives using CFD is becoming a realistic approach. ${ }^{4-6)} \mathrm{It}$ is expected that CFD plays an important role in advancing integrated design based on correct flight dynamic characteristics from the initial stage of design of advanced aircraft such as supersonic transport or blended wing-body aircraft, as well as today's matured commercial aircraft configuration. In Japan, JAXA renewed a dynamic stability test apparatus by introducing the forced excitation method in $2004^{3)}$ for the purpose of acquiring the transonic speed characteristics of the space transport HOPE-X, and the measurement accuracy was verified using the Standard Dynamics Model (SDM). As a corresponding CFD analysis for the SDM, unsteady calculation using JAXA's CFD code FaSTAR has been carried out and reported recently. ${ }^{6}$ )

The time-marching (TM) method in CFD can be used for any unsteady problem, but special care to maintain the same time accuracy is necessary to ensure the reliability of the solution. Although the authors have calculated dynamic coupled oscillation problems such as wing rock or flutter

(C) 2019 The Japan Society for Aeronautical and Space Sciences

*Received 11 August 2018; final revision received 4 February 2019; accepted for publication 13 June 2019.

†Corresponding author, miyaji@ynu.ac.jp boundary prediction, ${ }^{7)}$ the TM method with an appropriate time step requires a calculation time several tens of times that of the steady-flow method. In the small amplitude oscillation test for aircraft dynamic stability analysis, however, the flow field is expected to be similar to the harmonic vibration solution around the stationary solution. It is important to be able to efficiently perform a large number of analyses of such flows during aircraft design. The objective of this research is to develop a time spectral (TS) method, instead of using the TM method, that enables unsteady solutions to be obtained periodically, with an emphasis on predicting the dynamic stability derivatives of the aircraft.

The TS method was used in CFD for the first time by Hall et al. ${ }^{8)}$ to analyze the vibrational flow of a two-dimensional turbine blade cascade. It is also called the nonlinear frequency domain method or harmonic balance method. $\left.{ }^{9}, 10\right)$ New governing equations are derived for the coefficients of the time Fourier series expansion of the original unknown variables, and the solutions (expansion coefficients) are obtained as a converged solution with respect to the pseudotime. The TS method was applied to the aircraft stability problem by Murman, ${ }^{5)}$ and the dynamic derivatives of SDM were calculated using inviscid flow analysis on adaptively refined Cartesian grids. Moreover, Da Ronch et al. ${ }^{10)}$ also developed a TS method for RANS analysis on multiblock structured grids to evaluate the dynamic stability of large-sized passenger aircraft.

In order to enhance the efficiency and versatility of the TS method for simultaneous acquisition of static/dynamic stability derivatives, in this paper we introduce a fully implicit method for fast convergence of periodic unsteady RANS solutions on unstructured grids in pseudo-time. To the authors' knowledge, this is the first report on using the TS method for high-Reynolds number flows on unstructured grids handling arbitrary aircraft configurations. The transport 
equation for the turbulence model is also solved using the TS method. It is an extension of our previous report using structured grids, ${ }^{11)}$ in which we showed simple implementations using existing implicit TM code and succeeded in reducing the computation time by a factor of 15 for periodic flows. The procedure for the acquisition of stability derivatives is made more efficient using an automatic grid generator, HexaGrid, developed by JAXA. ${ }^{12)}$

\section{Numerical Algorithm}

\subsection{Governing equations in the time domain}

The governing equations are compressible Navier-Stokes equations with a moving boundary. The time-dependent equation in the integral form for the time-marching method is expressed as follows,

$$
\frac{\partial}{\partial t} \int_{\Omega} Q d V+\oint_{\partial \Omega(t)} F_{n} d S=0
$$

where, $Q$ is a conservative variable vector, $\Omega$ is the control volume (CV) around each node (vertex) of the grids, and $F_{n}$ is a flux vector in the normal direction to the control volume $(\mathrm{CV})$ boundary $\partial \Omega(t)$. In this study, the computational grids rotate rigidly, and then $\Omega$ is constant with time while the normal vector of the CV boundary $\partial \Omega(t)$ varies with time. We also use the Spalart-Allmaras turbulence model to calculate the eddy viscosity. More specifically, a model that considers quadratic constitutive relation $(\mathrm{QCR})^{13)}$ is used as it is known to be effective for suppressing excessive flow separation near the wing-body conjunction. The governing equation for the model is written in the same form as Eq. (1), with a source term added on the right-hand side.

\subsection{Spatial discretization}

A finite volume method is used for the spatial discretization. In Eq. (1), the CV average of the conservative variable in the first term is redefined as $Q$, and the second term (and the source term for the turbulence model) is expressed as $R(Q)$ to obtain the following semi-discretized equation,

$$
\frac{\partial(V \cdot Q)}{\partial t}+R(Q)=0
$$

where, $V$ is the volume of $\mathrm{CV}$. Since all of the computational grids move rigidly in this study to represent the motion of the object, the volume does not change over time. The highresolution upwind scheme SHUS is used for the numerical flux of the convective term and second-order accuracy in space is achieved by linear reconstruction of the primitive variables using Venkatakrishnan's slope limiter. Discretization of the viscous term corresponds to the second-order central scheme.

\subsection{Time spectral (TS) method}

The time spectral method is described below. If the solution vector $Q$ and residual $R(Q)$ are the periodic function of time, their finite Fourier series expansions are given by the following equation,

$$
\begin{aligned}
& Q(x, n \Delta t)=\sum_{k=-(N-1) / 2}^{(N-1) / 2} \hat{Q}_{k}(x) e^{i k \omega n \Delta t} \\
& R(x, n \Delta t)=\sum_{k=-(N-1) / 2}^{(N-1) / 2} \hat{R}_{k}(x) e^{i k \omega n \Delta t}
\end{aligned}
$$

where, $\hat{Q}_{k}$ and $\hat{R}_{k}$ are the complex Fourier coefficients, and $i$ is an imaginary unit. $N$ is the number of samples in one cycle $T$ (i.e., period of forced oscillation) and defined as $N=T / \Delta t$ with the sample interval time $\Delta t . N$ is an odd number in this study. The equation $\omega=2 \pi /(N \Delta t)$ is the angular frequency and $n$ denotes the data number $(n=-(N-1) / 2, \cdots 0$, $\cdots(N-1) / 2)$ in one cycle. In this study, $n=0$ at the forced oscillation phase $\phi=0$ of the object, and the range $-\pi<\phi<\pi$ is equally divided by $N$. Since $Q$ and $R$ are real numbers, the Fourier coefficient of the negative wave number $k$ is a complex conjugate of a corresponding positive wave number and the Fourier series is truncated by the $(N-1) / 2$-th harmonics of the frequency $\omega$ in Eqs. (3) and (4). Substituting these into Eq. (2) and using the orthogonality of the Fourier mode, the following equation is obtained.

$$
V \cdot i k \omega \hat{Q}_{k}+\hat{R}_{k}=0
$$

A pseudo-time term $V \cdot d \hat{Q}_{k} / d \tau$ is actually added to the left-hand side of Eq. (5) and Fourier coefficients are obtained as a steady solution. It could be solved explicitly with respect to $\tau$ with the use of Fourier/inverse Fourier transformations in the solution process. But it is difficult to directly apply the implicit time method since $\hat{R}_{k}$ is a function of $Q$ through $R$, and thus both $Q$ and $\hat{Q}_{k}$ appear in Eq. (5). Therefore, Eq. (5) is changed to include time-domain variables simply by substituting the discrete Fourier transform:

$$
\begin{aligned}
& \hat{Q}_{k}(x)=\frac{1}{N} \sum_{n=-(N-1) / 2}^{(N-1) / 2} Q(x, n \Delta t) e^{-i k \omega n \Delta t} \\
& \hat{R}_{k}(x)=\frac{1}{N} \sum_{n=-(N-1) / 2}^{(N-1) / 2} R(x, n \Delta t) e^{-i k \omega n \Delta t}
\end{aligned}
$$

By arranging this into real and imaginary parts, the following equations are finally obtained,

$$
V \frac{d Q_{n}}{d \tau}+V \omega \sum_{j=-(N-1) / 2}^{(N-1) / 2} c_{n j} Q_{j}+R_{n}=0
$$

where, $Q_{n}=Q(x, n \Delta t)$. The coefficient $c_{n j}$ is defined below.

$$
c_{n j}= \begin{cases}\frac{(-1)^{(n-j)}}{2} \operatorname{cosec}\left[\frac{\pi(n-j)}{N}\right] & (n \neq j) \\ 0 & (n=j)\end{cases}
$$

An implicit method for the pseudo-time is written as

$$
\begin{aligned}
& \left(\frac{I}{\Delta \tau} V+\frac{\partial R}{\partial Q}\right) \Delta Q_{n}+V \omega \sum_{j} c_{n j} \Delta Q_{j} \\
& =-\left(V \omega \sum_{j} c_{n j} Q_{j}+R_{n}\right) .
\end{aligned}
$$


The system of equation for $\Delta Q_{n}$ of all data numbers (i.e., all sample solutions in one cycle) is solved using the symmetric Gauss-Seidel method. By setting the initial value of $\Delta Q_{n}$ to 0 , and shifting the second term on the left-hand side to the right-hand side (diagonal term $c_{n j}=0$ ), it is solved using the forward/backward substitution below.

Forward:

$$
\begin{aligned}
\Delta Q_{n}^{*}= & \left(\frac{I}{\Delta \tau} V+\frac{\partial R}{\partial Q}\right)^{-1} \\
& \times\left[-\left(V \omega \sum_{j} c_{n j} Q_{j}+R_{n}\right)-V \omega \sum_{j<n} c_{n j} \Delta Q_{j}^{*}\right]
\end{aligned}
$$

Backward:

$$
\Delta Q_{n}=\Delta Q^{*}-\left(\frac{I}{\Delta \tau} V+\frac{\partial R}{\partial Q}\right)^{-1}\left[V \omega \sum_{j>n} c_{n j} \Delta Q_{j}\right]
$$

For inverting the (huge) coefficient matrix of the diagonal term, the same Matrix-free Gauss-Seidel method ${ }^{14)}$ as the TM method is used.

\section{Calculation Method for Dynamic Stability Derivatives}

Dynamic stability derivatives in the longitudinal motion are calculated as follows. Assuming that the pitching moment coefficient $C_{m}$ at a certain Mach number is a function of the angle-of-attack $\alpha$, pitch angular velocity $q=\dot{\theta}$, and their time derivatives $\dot{\alpha}$ and $\dot{q}$, the linearized equation of motion of an aircraft about the equilibrium state is written as follows,

$$
\begin{aligned}
I_{y y} \ddot{\theta}= & \frac{\rho U^{2} S \bar{c}}{2} \\
& \times\left(C_{m 0}+C_{m_{\alpha}} \Delta \alpha+\frac{l}{U} C_{m_{\dot{\alpha}}} \dot{\alpha}+\frac{l}{U} C_{m_{q}} q+\left(\frac{l}{U}\right)^{2} C_{m_{\dot{q}}} \dot{q}\right)
\end{aligned}
$$

where, $I_{y y}$ is the moment of inertia around the pitch axis, $\rho U^{2} / 2$ is the dynamic pressure, and $l$ is the reference length, which is one-half the size of the mean aerodynamic chord (MAC) $\bar{c}$. The non-dimensional stability derivatives are defined as follows,

$$
\begin{gathered}
C_{m_{\alpha}}=\frac{\partial C_{m}}{\partial \alpha}, \quad C_{m_{\dot{\alpha}}}=\frac{\partial C_{m}}{\partial\left(\frac{\dot{\alpha} \bar{c}}{2 U}\right)}, \\
C_{m_{q}}=\frac{\partial C_{m}}{\partial\left(\frac{q \bar{c}}{2 U}\right)}, \quad C_{m_{\dot{q}}}=\frac{\partial C_{m}}{\partial\left(\frac{\dot{q} \bar{c}^{2}}{4 U^{2}}\right)}
\end{gathered}
$$

By setting $\alpha=\theta$ and $\Delta \theta=\Delta \alpha=\alpha_{0} \sin (\omega t)$, the stability derivatives on the right-hand side of Eq. (13) are expressed as,

$$
\begin{aligned}
\Delta C_{m} & =C_{m}-C_{m 0} \\
& =\alpha_{0} k\left(C_{m_{\dot{\alpha}}}+C_{m_{q}}\right) \cos (\omega t)+\alpha_{0}\left(C_{m_{\alpha}}-k^{2} C_{m_{\dot{q}}}\right) \sin (\omega t)
\end{aligned}
$$

where, $k=\omega \bar{c} / 2 U$ is a reduced (non-dimensional) frequency. The physical meaning of each term in Eq. (15) can be seen by assuming the motion around a zero angle-of-attack:

$$
\Delta C_{m}=\frac{\bar{c}}{2 U}\left(C_{m_{q}}+C_{m_{\dot{\alpha}}}\right) \dot{\theta}+\left(C_{m_{\alpha}}-k^{2} C_{m_{\dot{q}}}\right) \theta
$$

Then, Eq. (13) can be expressed as,

$$
I_{y y} \ddot{\theta}-\frac{\bar{M} \bar{c}}{2 U}\left(C_{m_{q}}+C_{m_{\dot{\alpha}}}\right) \dot{\theta}-\bar{M}\left(C_{m_{\alpha}}-k^{2} C_{m_{\dot{q}}}\right) \theta=0
$$

where, $\bar{M}=\rho U^{2} S \bar{c} / 2$. In the above equation, the second term on the left-hand side represents the damping aerodynamic moment, and the third term represents the restoring moment. The term $C_{m_{q}}+C_{m_{\dot{\alpha}}}$ is referred to as the damping in pitch, while the term $C_{m_{\alpha}}-k^{2} C_{m_{\dot{q}}}$ is referred to as the oscillatory longitudinal stability. They are also called the outof-phase component and in-phase component ${ }^{4)}$ to the reference (forced) oscillation, respectively. In the TS method, these derivatives are obtained as the real and imaginary parts of the first-harmonic Fourier coefficients of $C_{m}$.

The dynamic derivative $C_{m_{\dot{\alpha}}}$ is separately obtained using the forced-heaving motion of an aircraft in the following way, although results are not included in this paper due to the lack of reference experiments. In the periodic heaving motion expressed as $z=\left(h_{0} \cdot l\right) \sin (\omega t)$, where $h_{0}$ is the non-dimensional amplitude normalized by $l=\bar{c} / 2$, the variation of $C_{m}$ in time is expanded as

$$
\begin{aligned}
\Delta C_{m} & =C_{m_{\alpha}} \Delta \alpha+\frac{l}{U} C_{m_{\dot{\alpha}}} \dot{\alpha} \\
& =\frac{h_{0} l \omega}{U} C_{m_{\alpha}} \cos (\omega t)-\frac{h_{0} l^{2} \omega^{2}}{U^{2}} C_{m_{\dot{\alpha}}} \sin (\omega t) \\
& =h_{0} k C_{m_{\alpha}} \cos (\omega t)-h_{0} k^{2} C_{m_{\dot{\alpha}}} \sin (\omega t)
\end{aligned}
$$

since $\Delta \alpha=\dot{z} / U, \dot{\alpha}=\ddot{z} / U$, and $q=\dot{q}=0$ in a similar formula found in Eq. (13). $C_{m_{\dot{\alpha}}}$ is obtained as the imaginary part of the first-harmonic Fourier coefficients of $C_{m}$ using the heaving motion.

\section{Results and Discussions}

Simulation results for two objects are shown in this section: one is the forced-pitch oscillation of a supercritical wing alone to compare unsteady pressure distribution with the experiment, and the other is the pitching oscillation for a simplified whole aircraft model to compare the stability derivatives at a wide range of Mach numbers. The pitching motions in both cases are described as follows,

$$
\alpha(t)=\alpha_{\mathrm{m}}+\alpha_{0} \sin (\omega t)
$$

where, $\alpha_{\mathrm{m}}$ and $\alpha_{0}$ denote the mean angle-of-attack and the amplitude of the forced oscillation, respectively. 


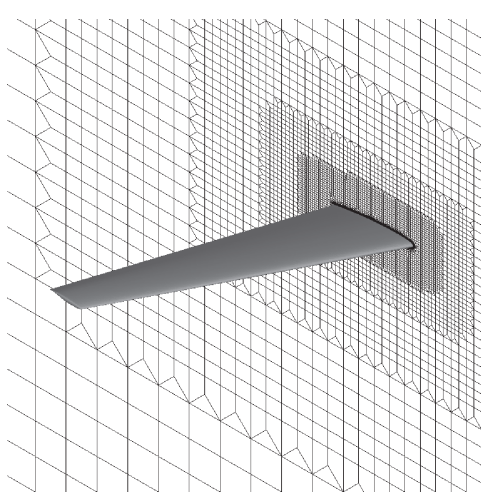

Fig. 1. Computational grids around the LANN wing (816,728 points).

Table 1. Flow conditions for the LANN wing.

\begin{tabular}{ccccc}
\hline$M_{\infty}$ & $\alpha_{\mathrm{m}}[\mathrm{deg}]$ & $\alpha_{0}[\mathrm{deg}]$ & $k$ & $R e$ \\
\hline 0.82 & 0.6 & 0.5 & 0.102 & $5.43 \times 10^{6}$ \\
\hline
\end{tabular}

All computational grids are generated using the HexaGrid, ${ }^{12)}$ developed by JAXA.

\subsection{LANN wing}

In this subsection, simulation results for the forced-pitch oscillation of a practical transonic wing called LockheedGeorgia, Air force, NASA Langley, NLR (LANN) model ${ }^{15,16)}$ are shown. The wing aspect ratio is 7.92 , the sweepback angle of $25 \%$-chord lines is $25^{\circ}$, and the taper ratio is 0.4 . The airfoil is a supercritical wing with $12 \%$ thickness. The maximum installation angle at the root chord is linearly twisted down toward the tip and the twist angle is $4.8^{\circ}$. Figure 1 shows the wing shape and the computational grids in a symmetrical plane. The number of total grid points is 816,728 .

The forced-pitch oscillation is performed around the axis of the $62 \%$ root chord location. The freestream Mach number is 0.82 , which was intensively examined in the wind tunnel experiment. Calculation conditions for the parameters in Eq. (19) and the reduced frequency $k$ are summarized in Table 1 . The Reynolds number $R e=5.43 \times 10^{6}$ in the wind tunnel is based on a MAC of 0.268 [m].

Both TS and TM simulations were run in this case to validate the TS method developed. For the TM method, the wing started oscillating from the steady flow at $\alpha_{\mathrm{m}}$ angleof-attack and the calculation continued until four cycles were reached to obtain the periodic solution. For the TS method, the number of sample points $N$ in one cycle was set to five, which corresponds to taking the Fourier modes up to the second harmonics.

Figure 2 shows the pressure contours for the steady flow. Figures 3(a) and (b) show steady-state pressure coefficient $\left(C_{p}\right)$ plots at $20 \%$ and $47.5 \%$ spanwise stations, respectively. The two-step change on the upper surface in Fig. 3(a) corresponds the $\lambda$-shaped shock waves in Fig. 2. The two shock waves near the root merge to a single shock wave towards the wingtip and a single step of $C_{p}$ is observed in Fig. 3(b). Figures 3(c)-(f) show the unsteady results resulting from the

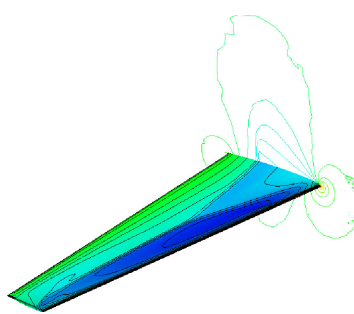

Fig. 2. Steady-state pressure distributions around the LANN wing at $M_{\infty}=0.82, R e=5.43 \times 10^{6}$, and 0.6-deg AOA

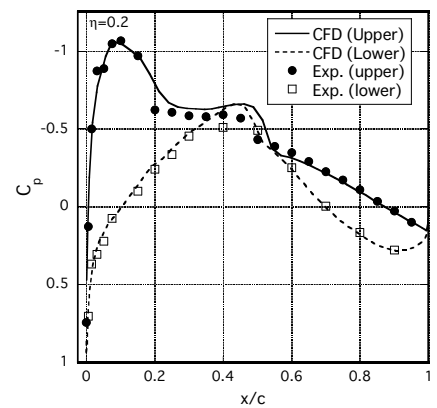

(a) Steady, $\eta=0.2$

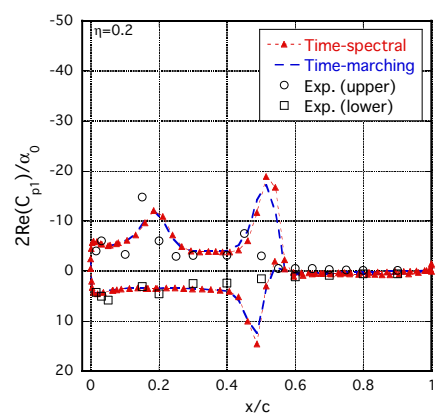

(c) $\operatorname{Re}\left(\hat{C}_{p_{1}}\right), \eta=0.2$

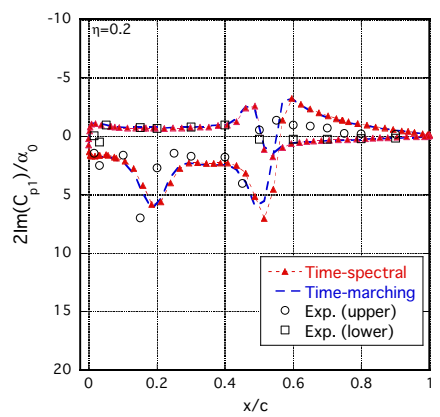

(e) $\operatorname{Im}\left(\hat{C}_{p_{1}}\right), \eta=0.2$

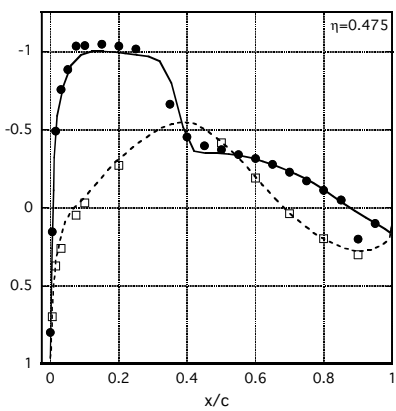

(b) Steady, $\eta=0.475$

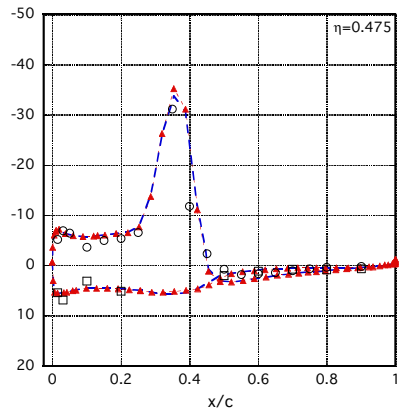

(d) $\operatorname{Re}\left(\hat{C}_{p_{1}}\right), \eta=0.475$

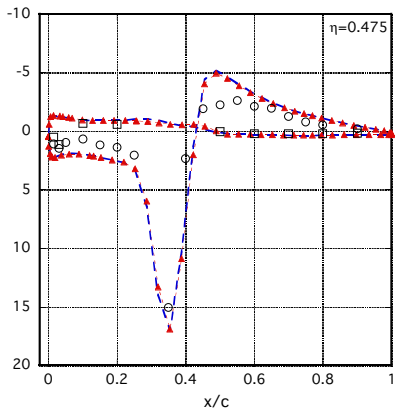

(f) $\operatorname{Im}\left(\hat{C}_{p_{1}}\right), \eta=0.475$
Fig. 3. Steady ((a), (b)) and first harmonics unsteady ((c)-(f)) surface $C_{p}$ distributions at two spanwise stations.

pitching motion, which are the real and imaginary parts of the Fourier coefficients' first harmonic component of the $C_{p}$ obtained as below.

$$
\begin{aligned}
C_{p}(t) & =\sum_{k=-\infty}^{\infty} \hat{C}_{p_{k}} e^{i k \omega t} \\
& =\hat{C}_{p_{0}}+2 \operatorname{Re}\left(\hat{C}_{p_{1}}\right) \cos (\omega t)-2 \operatorname{Im}\left(\hat{C}_{p_{1}}\right) \sin (\omega t)+\cdots
\end{aligned}
$$


The values in Figs. 3(c)-(f) are normalized by the amplitude $\alpha_{0}$ in radian. The TS and TM results agree with each other in all plots. Multiple peaks at $20 \%$ station and single peak at $47.5 \%$ station on the upper surface are the result of the shock-wave motions discussed above. Agreement with the experiment is also good, especially near the mid-span station. The absolute value (amplitude) of the second Fourier mode of the pitching-moment coefficient is about $10 \%$ of that of the first mode. Next, it was confirmed that the sample number of 5 is sufficient for small-amplitude, forced oscillation used in this study.

For the TM method, the number of time steps set for one cycle is 5,000 and the Newton iteration per step to eliminate unsteady residual is 5 , based on a preliminary study. For the TS method, $N=5$ sampled solutions converge to periodic unsteady solutions in less than 4,000 steps. Computation using the TS method was about 15 times faster than the TM method. In our implementation, the 'strong boundary condition' for the wall was not optimum for the implicit TS method, though it worked well for the TM method. Using the TS method, all residuals, including boundary nodes, should be expressed in the delta form, as done in Eqs. (11) and (12). Accordingly, the weak boundary condition for finite volume was better suited, allowing for larger pseudo-time steps and faster convergence.

\subsection{Aircraft model with $45^{\circ}$ sweptback wings}

Experimental data of dynamic stability derivatives obtained using forced-pitch and yaw oscillations of a simple aircraft configuration were reported in Bielat and Wiley. ${ }^{2)}$ The focus of this study is longitudinal characteristics. The airfoil of a NACA65A005 is commonly used for the main wing and horizontal/vertical tail wings. The sweptback angle at one-quarter chord is also common at $45^{\circ}$ for all wings. The aspect ratios of the main wing, horizontal tail and vertical tail are 4, 3.5, and 1.23, respectively, and the taper ratios are $0.2,0.4$, and 0.4 , respectively. The fuselage consists of an ogive nose and the following circular-cylinder. The model plan view is shown in Fig. 4.

The forced-pitch oscillation described in Eq. (19) is performed at a MAC of approximately $25 \%$ of the main wing. Calculation conditions are summarized in Table 2. The Reynolds number of the wind tunnel experiment is $R e=$ $1.1 \times 10^{6}$ based on a MAC of $0.175[\mathrm{~m}]$.

Computational grids with different resolutions were prepared. The coarse grid consists of 2,276,435 points and the fine grid shown in Fig. 5 consists of 5,024,987 points. All of the Mach numbers in Table 2 are simulated on the coarse grid, and three cases at $M_{\infty}=0.95,0.98$ and 1.03 are calculated on the fine grid.

The TM simulation was carried out again only at $M_{\infty}=$ 0.95 on the coarse grid to compare the results obtained using the TS method. The calculation procedure for the TM method is the same as that described in Subsection 4.1, and the number of sample points $N$ in one cycle for the TS method is also five. Figure 6 shows the dynamic dependence of the normal force coefficient $C_{N}$ and the pitching moment coefficient $C_{m}$ on the angle-of-attack $\alpha$ at $M_{\infty}=0.95$. Al-

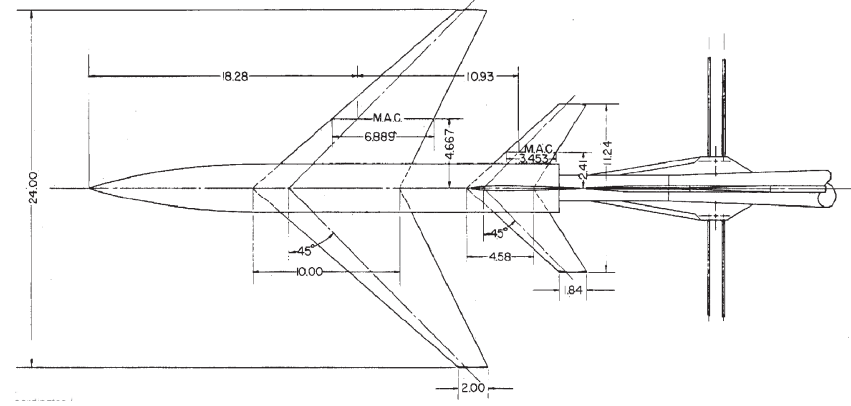

Fig. 4. Configuration of an aircraft model with $45^{\circ}$ sweptback wing and tails. $^{2)}$

Table 2. Flow conditions for an aircraft model.

\begin{tabular}{ccccc}
\hline$M_{\infty}$ & $\alpha_{\mathrm{m}}[\mathrm{deg}]$ & $\alpha_{0}[\mathrm{deg}]$ & $k$ & $R e$ \\
\hline $0.7,0.9,0.95,0.98,1.03,1.15$ & 0 & 2 & 0.024 & $1.1 \times 10^{6}$ \\
\hline
\end{tabular}

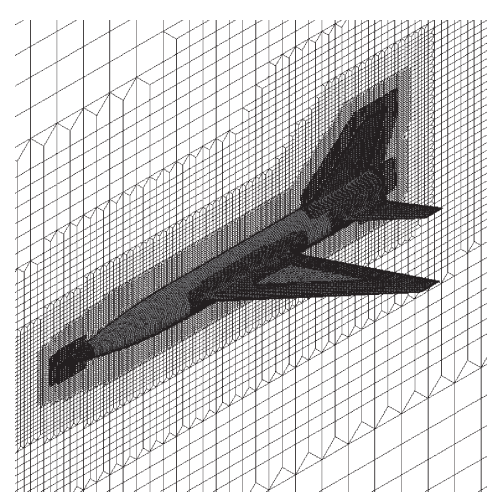

Fig. 5. Computational grids around an aircraft model (fine grids: $5,024,987$ points).

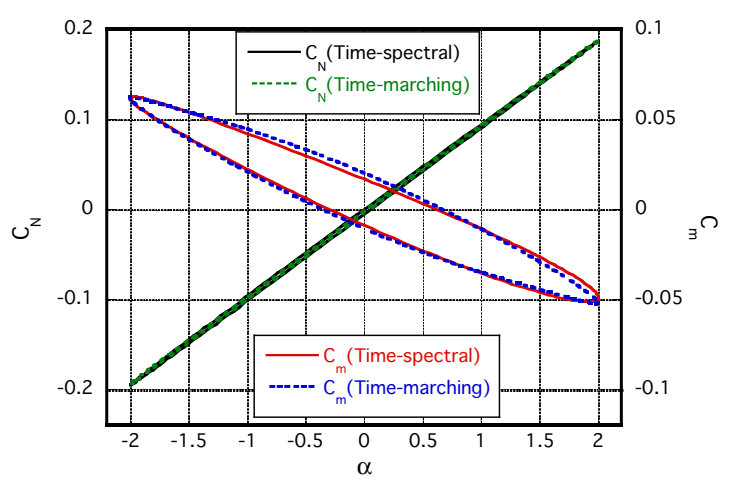

Fig. 6. Comparison of time-spectral and time-marching results for dynamic $C_{N}-\alpha$ and $C_{m}-\alpha$ at $M_{\infty}=0.95$.

most the same solutions are obtained with a shorter calculation time using the TS method. The hysteresis of $C_{N}$ is hardly visible compared to that of $C_{m}$. The profile of $C_{m}$ is close to an inclined ellipse, which shows that the influence of the third or higher harmonic components neglected by $N=5$ is small.

Figures 7(a) and (b) respectively show the responses of $C_{N}$ and $C_{m}$ for all of the Mach numbers on the coarse grid. As the Mach number increases, the slope of $C_{N}$ and $C_{m}$ in- 


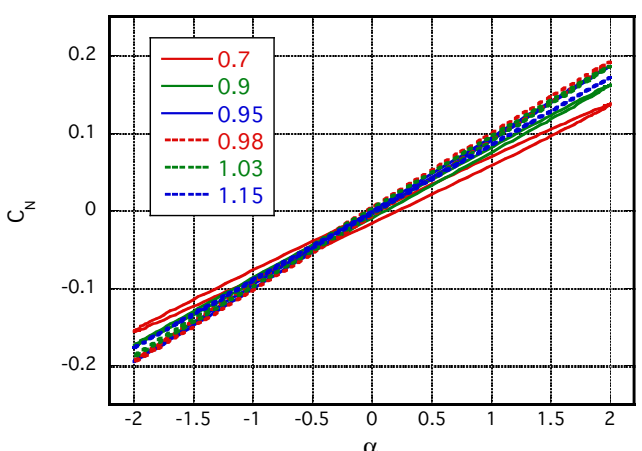

(a) $C_{N}-\alpha$

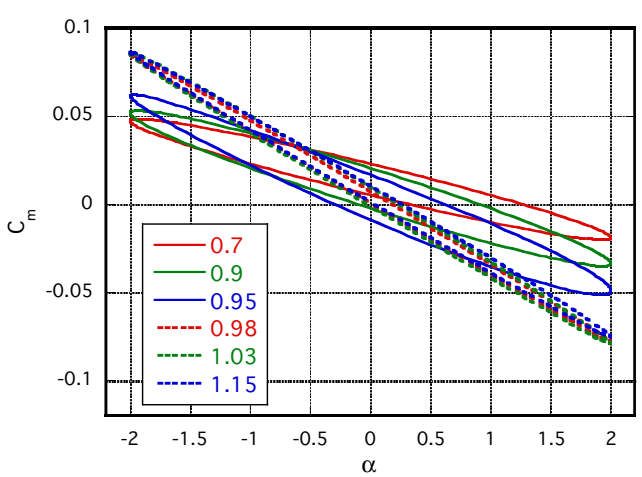

(b) $C_{m}-\alpha$

Fig. 7. Hysteresis of normal forces and pitching moments.

creases, and the difference between pitch-up and pitch-down at the same angle-of-attack becomes small (i.e., hysteresis decreases). We note that the absolute value (i.e., amplitude) of the second Fourier mode of $C_{m}$ is less than $3.5 \%$ of the first mode for all cases, thus validating the solution sample number of five. From these responses, the pitch damping $C_{m_{q}}+C_{m_{\dot{\alpha}}}$ and the oscillatory longitudinal stability $C_{m_{\alpha}}-k^{2} C_{m_{\dot{q}}}$ described in Section 3 are calculated and shown in Fig. 8. Although there is insufficient quantitative agreement, important features are reasonably captured. Regarding pitch damping, the tendency of abrupt change indicating a decrease in dynamic stability near $M_{\infty}=1$ is clearly shown. As for the oscillatory longitudinal stability, the tendency of gradual increase across $M_{\infty}=1$ is reproduced. The results on the fine grid at $M_{\infty}=0.95,0.98$, and 1.03 are also shown in Fig. 8, showing the effect of grid resolution. The stability derivatives values predicted using the fine grid are similar to those obtained using the coarse grid. The Mach number of abrupt change for pitch damping is again between $M_{\infty}=0.95$ and 0.98 , which is slightly lower than the experimental results. We further investigated the effect of the outer boundary location and the effect of viscosity on the results. In the latter case, simulations were run on completely different unstructured grids composed of isotropic tetrahedron elements. No major differences were observed in these tests. Considering the variance for each wind tunnel test observed in the dynamic test of $\mathrm{SDM}^{1,3)}$ and similarly quantitative differences found in another study, ${ }^{5)}$ we regard our results as reasonably accurate.

The characteristic change in stability derivatives shown

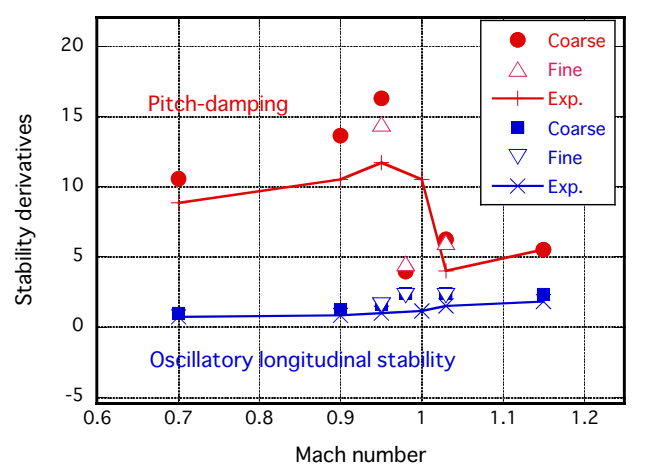

Fig. 8. Variation in pitch-damping and oscillatory longitudinal stability derivatives due to the flow Mach number.

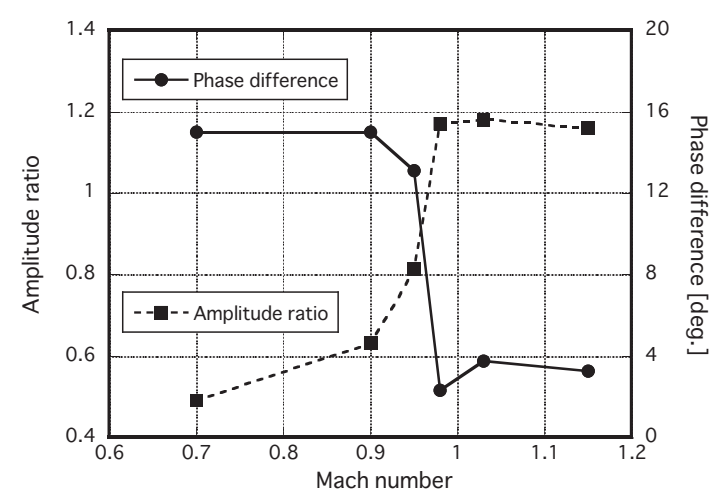

Fig. 9. Variation in the amplitude ratio and the phase difference of $C_{m}$ against forced-pitch oscillation.

above can be explained by the Mach number dependencies in Fig. 7(b). First, the increase in the negative slope of $C_{m}$ with the increasing Mach number corresponds to the increase in (oscillatory) longitudinal stability in Fig. 8. Next, since all of the ellipse-like history in Fig. 7(b) transits counterclockwise, the area enclosed denotes the kinetic energy of the aircraft lost due to fluid flow during one cycle. The decrease in the area as the Mach number increases then reflects the decrease in pitch damping shown in Fig. 8. Figure 9 additionally supports this explanation, which shows the input (i.e., forced sinusoidal pitching)-to-output (i.e., response of $C_{m}$ ) relation. In the two ordinates, the amplitude ratio denotes $\left|\hat{C}_{m_{1}}\right| / \alpha_{0}$ and the phase difference denotes $\arg \left(\hat{C}_{m_{1}}\right)$. They respectively correspond to the magnitude of the oscillatory longitudinal stability and pitch damping. The plots show the same Mach number dependency as Fig. 8.

The flows for $M_{\infty}=0.95$ and 1.03 showing significant differences in damping are compared. Figures 10(a)-(d) show instantaneous surface pressure distributions at the phase of $\phi=0$ and $2 \pi / 5$, which are respectively $n=0$ and 1 in terms of the data number among $N=5$ samples in the cycle. The pressure contours in the MAC spanwise section are also shown. In the case of $M_{\infty}=0.95$ in Figs. 10(a) and (b), the shock wave on the main wing is almost perpendicular to the freestream, and is located at a MAC of approximately $60 \%$ to $70 \%$. In the case of $M_{\infty}=1.03$ in Figs. $10(\mathrm{c})$ and (d), the shock wave is parallel 


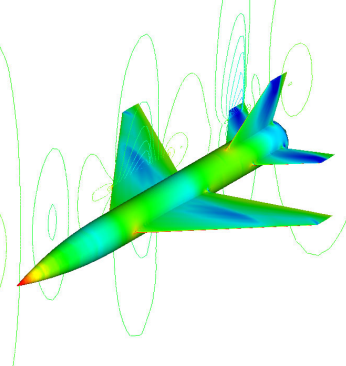

(a) $M_{\infty}=0.95, \phi=0$

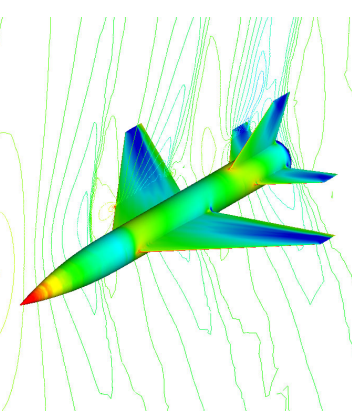

(c) $M_{\infty}=1.03, \phi=0$

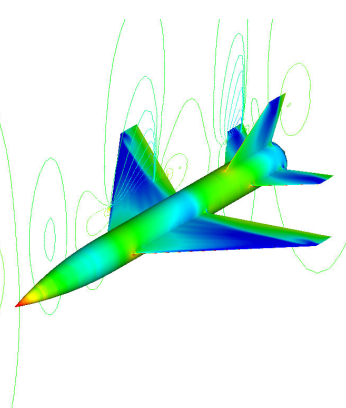

(b) $M_{\infty}=0.95, \phi=2 \pi / 5$

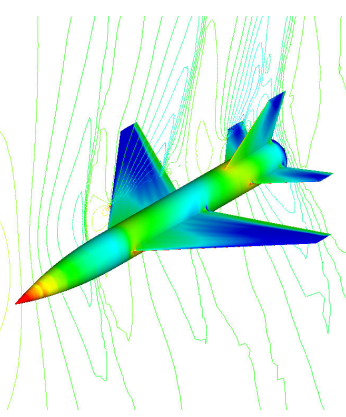

(d) $M_{\infty}=1.03, \phi=2 \pi / 5$
Fig. 10. Instantaneous surface pressure distributions.

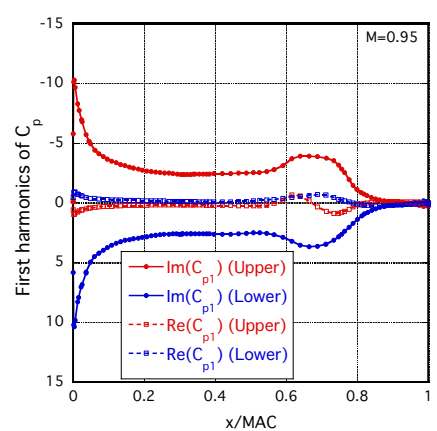

(a) Main wing at $M_{\infty}=0.95$

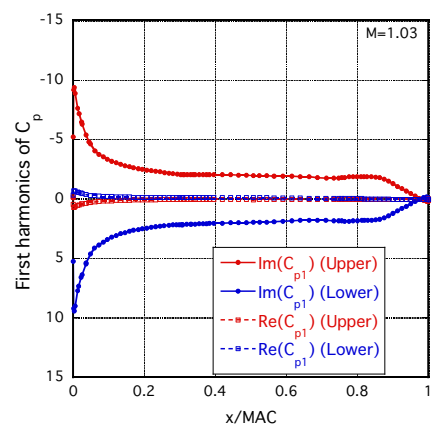

(c) Main wing at $M_{\infty}=1.03$

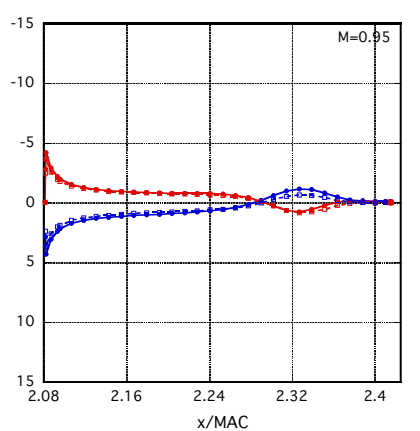

(b) Tail wing at $M_{\infty}=0.95$

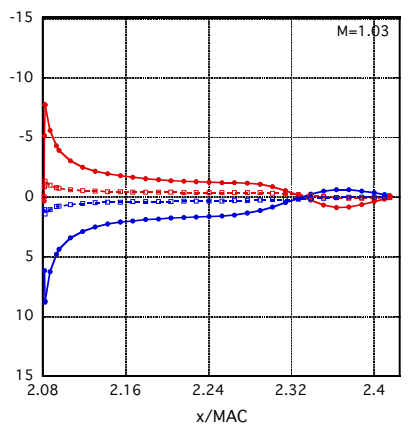

(d) Tail wing at $M_{\infty}=1.03$
Fig. 11. Real and imaginary parts of the first harmonic of unsteady surface $C_{p}$ distributions at $M_{\infty}=0.95$ and 1.03 .

to the trailing-edge near $90 \%$ MAC. The real and imaginary parts of the first harmonic component of unsteady $C_{p}$ at the MAC section are shown in Figs. 11(a)-(d). Figures 11(a) and (b) are those at $M_{\infty}=0.95$ on the main wing and tail wing, respectively. Figures 11(c) and (d) are the same sets at $M_{\infty}=1.03$. Figures 12 (a) and (b) further show the sur-

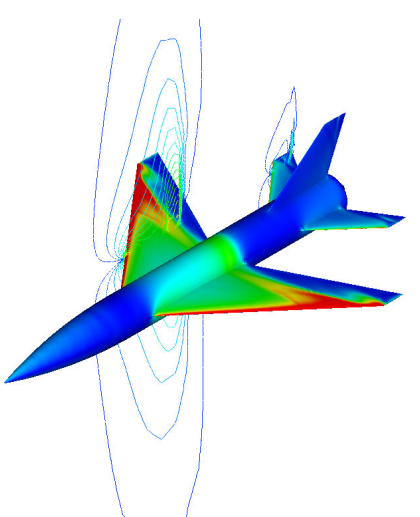

(a) $M_{\infty}=0.95$

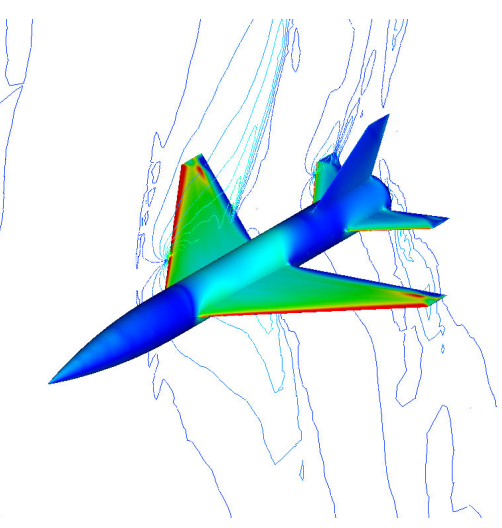

(b) $M_{\infty}=1.03$

Fig. 12. Amplitude $\left(\left|\hat{C}_{p_{1}}\right|\right)$ of the first harmonics of unsteady $C_{p}$.

face distributions of the first harmonics magnitude, $\left|\hat{C}_{p_{1}}\right|$. In Figs. 11(a) and (c), the imaginary part, or the in-phase component of $C_{p}$, is dominant on the main wing at both Mach numbers. Another important feature is a clear peak in the imaginary part from $60 \%$ to $75 \%$ chord in Fig. 11(a), in contrast to almost flat distribution in Fig. 11(c). These features are also seen in the surface plots in Figs. 12(a) and (b). The region of large amplitude of unsteady $C_{p}$ exists in Fig. 12(a) on the main wing from the mid-span to tip region, which is due to the shock-wave motion. While in Fig. 12(b), the amplitude on the main wing is almost uniform except for the leading-edge and trailing-edge. As for the horizontal tail wing, the real and imaginary parts have similar amplitudes at $M_{\infty}=0.95$ in Fig. 11(b), which indicates the phase difference from the forced oscillation. The imaginary part at $M_{\infty}=1.03$ in Fig. $11(\mathrm{~d})$ is again dominant, like the main wing. The clear peak due to the shock-wave motion over the main wing and the phase difference on the tail wing at $M_{\infty}=0.95$ result in the phase difference in the $C_{m}$ response discussed in Figs. 7 to 9.

\section{Conclusion}

A time-spectral (TS) CFD method for RANS simulation on unstructured grids has been developed to efficiently analyze the periodic unsteady flow around a oscillating object. Using the conventional time-marching (TM) method, special attention is required to obtain a solution that does not depend 
on the time-step size, while in the TS method, sampled solutions in one cycle are obtained as steady solutions in the pseudo-time. The method is validated through two problems: One is for predicting unsteady pressures on an oscillating transonic wing, and the other is for predicting dynamic stability derivatives of a simple whole aircraft configuration. In both cases, the TS results agreed with the TM results, doing so while requiring a computation time that is shorter by a factor of approximately 4 . The dependencies of the aircraft dynamic stability on the flow Mach number were captured well, although quantitative agreement with the experiment was not sufficient. The effectiveness and accuracies of the method will be further verified for a wider range of calculation objects and calculation conditions.

\section{Acknowledgments}

This work was supported by JSPS KAKENHI Grant Number JP19K04835.

The authors wish to express their gratitude to JAXA for providing the automatic grid generator, HexaGrid.

\section{References}

1) Kalviste, J.: Use of Rotary Balance and Forced Oscillation Test Data in a Six Degrees of Freedom Simulation, AIAA Paper 82-1364, 1982.

2) Bielat, R. P. and Wiley, H. G.: Dynamic Longitudinal and Directional Stability Derivatives for a $45^{\circ}$ Sweptback-Wing Airplane Model at Transonic Speeds, NASA TM X-39, 1959.

3) Miwa, H. and Ueno, M.: Development of Dynamic Stability Equipment for Transonic Wind Tunnel, JAXA-RR-03-021, 2004, pp. 1-49 (in Japanese).

4) Da Ronch, A., Vallespin, D., Ghoreyshi, M., and Badcock, K. J.: Evaluation of Dynamic Derivatives Using Computational Fluid Dynamics, AIAA J., 50, 2 (2012), pp. 470-484.

5) Murman, S. M.: Reduced-Frequency Approach for Calculating Dy- namic Derivatives, AIAA J., 45, 6 (2007), pp. 1161-1168.

6) Hashimoto, A., Hashizume, M., Sunada, S., Murakami, K., and Ueno, M.: Steady/Unsteady Analysis of Aerodynamic Derivatives on Standard Dynamics Model, Proceedings of 44th Fluid Dynamics Conference/30th Aerospace Numerical Simulation Symposium, 2E07, June 2012 (in Japanese).

7) Miyaji, K., Machida, K., Fujita, K., and Sawai, S.: Tail-Wing Flutter Analyses of a Balloon-based Operation Vehicle for Microgravity Experiments, Trans. Jpn. Soc. Aeronaut. Space Sci., 55 (2012), pp. 333-340.

8) Hall, K. C., Thomas, J. P., and Clark, W. S.: Computation of Unsteady Nonlinear Flows in Cascades Using a Harmonic Balance Technique, AIAA J., 40, 5 (2002), pp. 879-886.

9) McMullen, M., Jameson, A., and Alonso, J. J.: Application of NonLinear Frequency Domain Solver to the Euler and Navier-Stokes Equations, AIAA Paper 2002-0120, 2002.

10) Da Ronch, A., McCracken, A. J., Badcock, K. J., Widhalm, M., and Campobasso, M. S.: Linear Frequency Domain and Harmonic Balance Predictions of Dynamic Derivatives, J. Aircraft, 50, 3 (2013), pp. 694-707.

11) Taniichi, K. and Miyaji, K.: Development of an Efficient TimeSpectral Method for Periodically Unsteady Flow Solutions, J. Jpn. Soc. Aeronaut. Space Sci., 65 (2017), pp. 73-81 (in Japanese).

12) Hashimoto, A., Murakami, K., Aoyama, T., and Lahur, P.: Lift and Drag Prediction Using Automatic Hexahedra Grid Generation Method, AIAA Paper 2009-1365, 2009.

13) Spalart, P. R.: Strategies for Turbulence Modeling and Simulation, Int. J. Heat Fluid Flow, 21 (2000), pp. 252-263.

14) Shima, E.: A Simple Implicit Scheme for Structured/Unstructured CFD, Proceedings of 29th Fluid Dynamics Conference, 2C9, Sep. 1997 (in Japanese).

15) Malone, J. B. and Ruo, S. Y.: LANN Wing Test Program: Acquisition and Application of Unsteady Transonic Data for Evaluation of Threedimensional Computational Methods, AFWAL-TR-83-3006, 1983.

16) Zwaan, R. J.: LANN Wing Pitching Oscillation, Compendium of Unsteady Aerodynamic Measurements, AGARD-R-702, Addendum No. 1, 1985, pp. 9-1-9-76.

Shigeru Saito Associate Editor 